CASE REPORT

D.W. Kim

S.H. Kim

S.J. Jung

\section{Successful Sonography-Guided Fine-Needle Aspiration Biopsy of a 1-Millimeter-Diameter Papillary Thyroid Microcarcinoma}

SUMMARY: A PTMC is defined as a thyroid papillary cancer that measures $\leq 10 \mathrm{~mm}$ in the greatest diameter. The detection rate for a PTMC has increased because of the worldwide use of highresolution sonography and US-FNAB. We report a case of a 1-mm-diameter PTMC confirmed at thyroidectomy for which the cytologic diagnosis had an important role in deciding the extent of surgery (ie, either a total thyroidectomy or a hemithyroidectomy).

ABBREVIATIONS: ATA = American Thyroid Association; HE = hematoxylin-eosin; PTC = papillary thyroid carcinoma; PTMC = papillary thyroid microcarcinoma; US-FNAB = sonography-guided fine-needle aspiration biopsy
A US-FNAB is a widely used procedure for the cytological evaluation of thyroid nodules. However, US-FNAB of a thyroid nodule $<10 \mathrm{~mm}$ in maximal diameter seems to be limited because of a poor clinical significance and social-medical-cost problem. We report a case of $1 \mathrm{~mm}$ diameter PTMC that was diagnosed on US-FNAB and this cytological diagnosis influenced the decision of thyroid surgery type.

\section{Case Report}

A 43-year-old woman who had recently complained of fatigue and neck discomfort was referred to the radiology department from the department of general surgery for sonographic evaluation of the thyroid. The patient showed normal values on a thyroid function test (T3, $1.3 \mathrm{nmol} / \mathrm{L}$; free T4, $11.6 \mathrm{pmol} / \mathrm{L}$; thyroid-stimulating hormone, $1.03 \mathrm{mU} / \mathrm{L})$, and no specific abnormalities were detected on a physical examination of the neck. The patient had neither previously undergone a fine-needle biopsy for a thyroid lesion nor had a history of neck irradiation or surgery or a family history of a thyroid malignancy.

A thyroid nodule that showed marked hypoechogenicity, an illdefined margin, and a taller-than-wide shape was identified in the left lobe on thyroid sonography. The nodule measured $5.5 \mathrm{~mm}$ in the maximal diameter and was considered to be highly suspicious for a malignancy (Fig 1A). A hypoechoic thyroid nodule that measured 1.0 $\mathrm{mm}$ in maximal diameter was detected in the medial aspect of the opposite right midlobe on a simultaneous diagnostic sonography examination, and this nodule was considered as an "indeterminate" nodule for a malignancy (Fig 1B). In addition, several thyroid nodules that showed benign sonographic features were observed in both lobes.

A US-FNAB was performed by 1 investigator (D.W.K.) on the 2 nodules that showed a likelihood of being thyroid malignancies. Only 1 sampling was achieved for each thyroid nodule, without the administration of local anesthetic. A US-FNAB was not performed on several other thyroid nodules suggestive of being benign lesions on thyroid sonography because the selection of a nodule for US-FNAB was

\section{Received April 14, 2009; accepted after revision July 25}

From the Departments of Radiology (D.W.K.), General Surgery (Thyroid and Breast Clinic) (S.H.K.), and Pathology (S.J.J.), Busan Paik Hospital, Inje University College of Medicine, Busan, South Korea

Please address correspondence to Dong Wook Kim, Department of Radiology, Busan Paik Hospital, Inje University College of Medicine, 633-165 Gaegeum-dong, Busanjin-gu, Busan, South Korea 614-734; e-mail: dwultra@lycos.co.kr

DOI 10.3174/ajnr.A1850 based primarily on the sonographic features rather than the nodule size. The patient had no significant pain or other complications following the US-FNAB procedure. On the basis of the cytology results, a nodule highly suspicious for a malignancy in the left lobe was identified as compatible with PTC (Fig 1C), and a 1-mm right-lobe nodule suspicious for a malignancy on thyroid sonography was also identified as suspicious for PTC (Fig 1D).

Although the primary PTC had a maximal diameter of $5.5 \mathrm{~mm}$ and an intraglandular location, the surgeon and the patient chose a total thyroidectomy rather than a left hemithyroidectomy on the basis of the cytology findings. On pathology, the tiny malignancy in the right thyroid lobe measured $1.5 \mathrm{~mm}$ in maximal diameter at gross and microscopic examinations. On the pathologic findings, PTMCs in left and right thyroid lobes were a classic type and follicular variant, respectively (Fig 1E, -F). These 2 PTMCs showed no capsular or nodal invasion in the perithyroidal areas. In addition, several thyroid nodules that had showed benign features on thyroid sonography in both lobes were determined to be nodular hyperplasia at microscopic findings. After thyroid surgery, the patient has undergone follow-up and has not had any problems related to the thyroid malignancy or the surgery.

\section{Discussion}

A PTMC is defined as a PTC $<10 \mathrm{~mm}$ in the maximal diameter. The detection and incidence rates of PTMCs have increased recently with the worldwide use of thyroid sonography and US-FNABs, and there have been many reports related to the diagnostic efficacy of US-FNAB for PTMCs. ${ }^{1-6}$ The ATA guidelines and the Society of Radiologists in Sonography Consensus Statement suggest that, in general, only nodules $>10$ $\mathrm{mm}$ in diameter should be evaluated because these nodules have the potential to represent a clinically significant cancer. ${ }^{7,8}$ However, both the ATA and Society of Radiologists in Sonography Consensus Statement suggest performing US-FNAB on thyroid nodules $\geq 10 \mathrm{~mm}$ in maximal diameter when the nodules exhibit sonographic features associated with thyroid cancer. Recently, Roti et $\mathrm{al}^{9}$ reported that this incidence of bilaterality of a PTMC is $19 \%$. This suggests that incomplete treatment would occur in 19\% of patients if only hemithyroidectomy was performed. Therefore, we believe that an accurate evaluation of satellite malignancy in an opposite thyroid lobe 

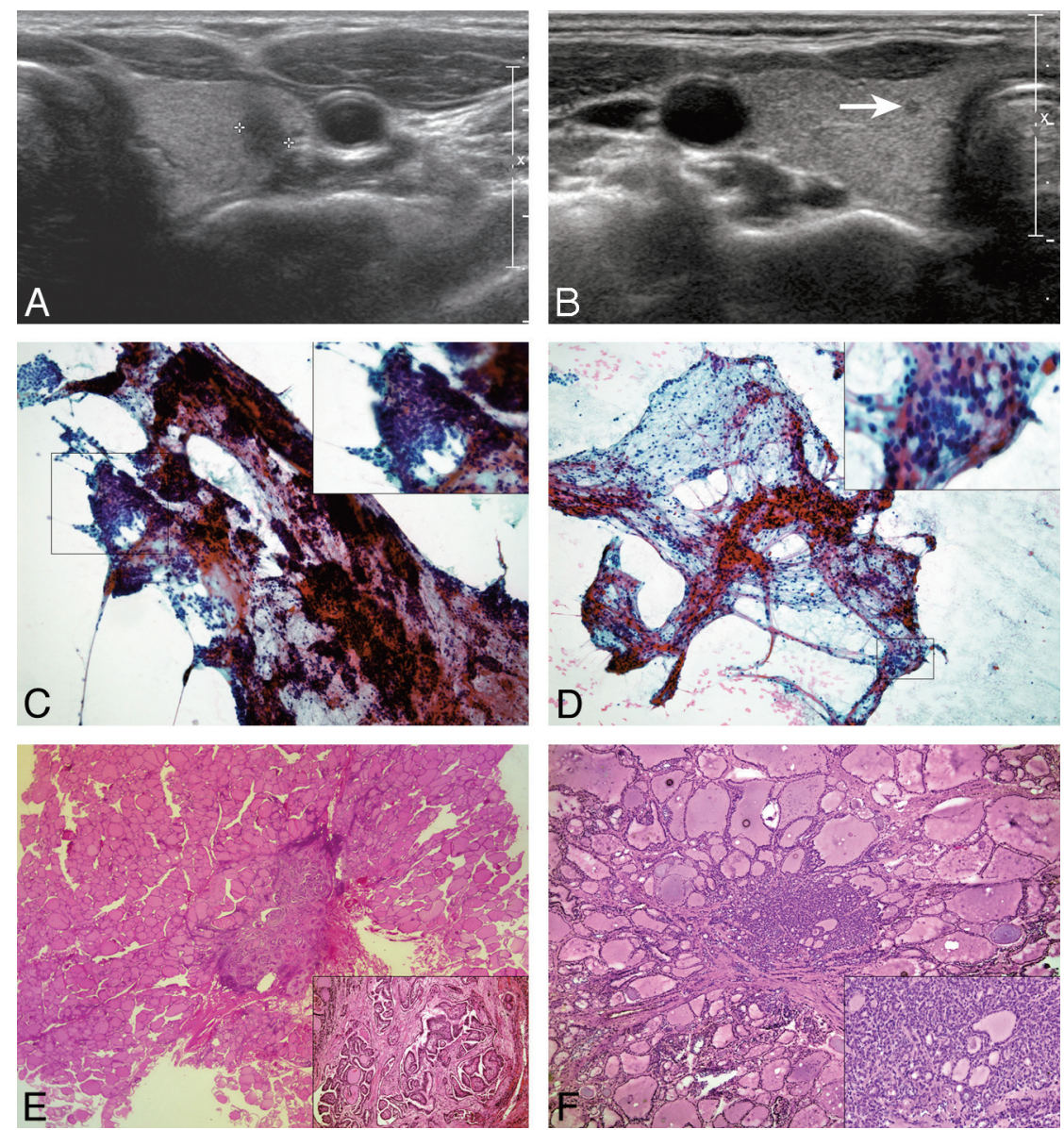

Fig 1. A, A transverse sonogram shows a 5.5-mm-diameter solid thyroid nodule (calipers) with marked hypoechogenicity, an irregular margin, and a taller-than-wide shape located in the left thyroid lobe. $B, A$ transverse sonogram shows a 1.0 -mm-diameter solid thyroid nodule with hypoechogenicity in the right thyroid lobe (arrow). $C$, On aspiration cytology of left thyroid nodule, many 3D papillary or caplike structured clusters are noted. The characteristic nuclear features of papillary carcinoma, including fine and powdery chromatin and irregular nuclear membranes with nuclear grooves, are noted in most nuclei (Papanicolaou, original magnification $\times 100$ [inset, $\times 400]$ ). D, Aspiration cytology of the right thyroid nodule reveals a few small aggregates of follicular cells within some lymphocytes. The follicular cells reveal nuclear clearing and grooves suspicious for papillary carcinoma (Papanicolaou, original magnification $\times 100$ [inset, $\times 400]$ ]. E, On higher magnification of left thyroid nodule, the papillary arrangement is well recognized and is considered a classic type of PTMC (HE, original magnification $\times 40$ [inset, $\times 100]$ ). F, Right thyroid nodule reveals a follicular variant of PTMC, which is composed of follicles having nuclear features of papillary carcinoma on a frozen-section slide (HE, original magnification $\times 10$ [inset, $\times 100])$.

through sonographic diagnosis and US-FNAB of the thyroid nodule is necessary.

The sampling technique for US-FNAB of small thyroid nodules $<5 \mathrm{~mm}$ in maximal diameter is the same as that for thyroid nodules $>5 \mathrm{~mm}$ in maximal diameter. After the needle tip is placed in the appropriate area of the target nodule, the sampling begins by using the "mixed sampling technique, "6,10 in which the needle is moved up and down for a few seconds only by movement of the operator's wrist and without initial aspiration under sonographic guidance. When the hub of the needle is filled with the proper amount of material, the syringe-needle unit should be rapidly withdrawn. However, if no aspirates are obtained after a few seconds of capillary sampling, negative pressure can gradually be added by use of the first finger of the right hand. This to-and-fro needle movement should be continued during the entire sampling procedure. Only 1 sampling is achieved in most of the US-FNABs for thyroid nodules by using 1 needle puncture and without local anesthesia.

The smear technique is as follows: The operator expels the contents of the needle hub onto 1 or 2 slides for smear preparation after filling the empty syringe with room air. Two-to-4 slides are then obtained by duplicating the smear. Finally, a special technique is used in which the operator flips the plastic hub of the needle onto the slide for effective cell acquisition of the remaining material in the needle hub. ${ }^{10}$

One of the investigators (D.W.K.) has performed USFNAB for small thyroid nodules $<5 \mathrm{~mm}$ in maximal diameter when a nodule has shown the likelihood of a malignancy on diagnostic thyroid sonography. ${ }^{6}$ In particular, US-FNAB has been performed for small thyroid nodules regardless of the nodule size when it is necessary to determine the type of thyroid surgery (a total thyroidectomy or hemithyroidectomy), if the primary thyroid malignancy is a PTC and is $<10 \mathrm{~mm}$ in maximal diameter. Our present patient had a small thyroid nodule in the left thyroid lobe that was considered highly suspicious for a malignancy as well as a tiny nodule in the right thyroid lobe that was considered indeterminate for a malignancy on thyroid sonography. The patient underwent a total thyroidectomy based on cytologic findings after a successful US-FNAB for each nodule.

In conclusion, we report a case of a 1-mm-diameter PTMC that was diagnosed by cytology after US-FNAB and confirmed after thyroidectomy. The use of US-FNAB for a 1-mm-diam- 
eter thyroid nodule influenced the decision regarding the most appropriate type of thyroid surgery technique.

\section{References}

1. Yokozawa T, Miyauchi A, Kuma K, et al. Accurate and simple method of diagnosing thyroid nodules: the modified technique of ultrasound-guided fine needle aspiration biopsy. Thyroid 1995;5:141-45

2. Leenhardt L, Hejblum G, Farnc B, et al. Indications and limits of ultrasoundguided cytology in the management of nonpalpable thyroid nodules. J Clin Endocrinol Metab 1999;84:242-48

3. Kim SJ, Kim EK, Park CS, et al. Ultrasound-guided fine-needle aspiration biopsy in nonpalpable thyroid nodules: is it useful in infracentimetric nodules? Yonsei Med J 2003;44:635-40

4. Nam-Goong IS, Kim HY, Gong GY, et al. Ultrasonography-guided fine-needle aspiration of thyroid incidentaloma: correlation with pathological findings. J Clin Endocrinol 2004;60:21-28
5. Burman KD. Editorial: micropapillary thyroid cancer-should we aspirate all nodules regardless of size? J Clin Endocrinol Metab 2006;91:2043-46

6. Kim DW, Lee EJ, Kim SH, et al. Ultrasound-guided fine-needle aspiration biopsy of thyroid nodules: comparison in efficacy according to nodule size. Thyroid 2009;19:27-31

7. Gharib H, Papini E, Valcavi R, et al. American Association of Clinical Endocrinologists and Associazione Medici Endorinologi medical guidelines for clinical practice for the diagnosis and management of thyroid nodules. Endocr Pract 2006;12:63-102

8. Frates MC, Benson CB, Charboneau JW, et al. Management of thyroid nodules detected at US: Society of Radiologists in Ultrasound consensus conference statement. Radiology 2005;237:794-800

9. Roti E, Rossi R, Tranforini G, et al. Clinical and histological characteristics of papillary thyroid microcarcinoma: results of retrospective study in 243 patients. J Clin Endocrinol Metab 2006;91:2171-78

10. Park KR, Kim DW, Rho MH, et al. Ultrasonography-guided fine-needle aspiration biopsy of thyroid nodule: effective technique and a peculiar smear method [In Korean]. J Korean Radiol Soc 2006;55:543-49 